\title{
Presidential Address to the American Society for Clinical Investigation, Washington DC, May 4, 1996 Is There Room Left for Academics in Academic Medicine?
}

\author{
Judith L. Swain \\ Cardiovascular Division, University of Pennsylvania Health System, Philadelphia, Pennsylvania 19104
}

The question that I have posed for consideration this afternoon is whether there is any longer room left for academics in academic medicine. I should say at the outset that I believe we can answer this question in the affirmative for now, but that to continue to do so, we may have to consider changing the structure of our training programs, and of our faculties. I would like to present some thoughts on how we can preserve the elements that we value as academic physicians. The structure that I will discuss is not novel, but rather currently exists in some form in number of academic medical centers. Because of time constraints I will discuss specific categories of trainees and faculty members, but in reality these groups should not be rigidly defined but rather should provide a focus for career development.

As we all know, the three cornerstones of our academic mission are education, patient care, and research. These three endeavors are interdependent, and excellence in all three areas is necessary. I won't reiterate the data to support the hard facts which we now see as self-evident, and which include the fact that reimbursement for patient care is decreasing, resulting in a decrease in the flexible dollars that have been used to support research and education; that there is increasing competition for both patients and research dollars; that entry of "for-profits" into academic medical centers is threatening our mission; and that "rightsizing" of many academic programs is now occurring so that the range of opportunities to enjoy a career in academic medicine is no longer expanding.

Much has been said concerning how academic medical centers can secure a patient base, so I won't address this challenge today. But even if we ensure that our academic medical centers have a well developed and successful plan for securing patients, there are many challenges in meeting our other two missions-research and education. I would like to discuss my thoughts on how we should train young people for a career in academic medicine, and what I believe the opportunities are, or will be, for attaining a satisfying and rewarding academic career in the future. By articulating what we expect of our trainees, and what our trainees should expect of faculty positions, we may be better able to address the challenge of keeping academics in academic medicine.

With respect to our training programs, I believe it is important to develop a mission statement that is subscribed to by all members of an academic program. A cornerstone of the mission must be that trainees no longer be considered essential for

Address correspondence to Judith L. Swain, M.D., Division of Cardiology, Hospital of University of Pennsylvania, Philadelphia, PA 19104. Phone: 215-662-3140; FAX: 215-349-8017.

Received for publication 12 August 1996.

J. Clin. Invest.

(C) The American Society for Clinical Investigation, Inc. 0021-9738/96/09/1071/03 \$2.00

Volume 98, Number 5, September 1996, 1071-1073 delivering patient care, but rather provide this service function in the context of an educational program. It is important that we avoid the statement "we need a resident or fellow on this service." Once we get to the point that clinical activities are independent of the presence of a trainee, then we have reached the point at which our training programs are targeted to developing our future academic physicians. It is also essential that we clearly articulate the expectations that we have for our trainees. It is important that trainees develop an academic theme. It is all too easy for trainees to focus on one rotation at a time, or one year of training at a time. It is important that the academic leadership plan individual programs for trainees that encompass a three to five year training period. In this way, at the end of the formal training program, that trainee will have a portfolio of accomplishments which will position him or her for a career in academic medicine.

We should apply a litmus test to our training programs. When a trainee successfully completes the proscribed training program, will he or she be the type of candidate who would be competitive for a faculty position in our own institution? It is most instructive to go through the process of faculty recruitment to identify the positive and negative characteristics of candidates, and then evaluate our own trainees in this context. If our own trainees don't measure up to the best from outside the institution, then we must reconsider the structure of our program.

Training programs must prepare trainees for the types of faculty positions that will exist in the future, and these faculty positions may be different than those in existence now. More rigorous and focused training pathways may better prepare trainees for careers in the academic medical center of the future. I will make an argument later that we really need four categories of faculty members in the future, and that we can develop these faculty members through three distinct types of training programs. I believe many of us would agree that we would not pretend to train future generations as we ourselves trained. Many of us, through a combination of luck and talent, have achieved successful careers in academic medicine. We need to design their training in a much more rigorous fashion to meet specific goals. There are three broad training tracks that I believe will position our young trainees to enter a career in academic medicine: the physician scientist training program; the clinical investigator training pathway; and the clinical educator pathway. Although virtually all academic faculty members participate in research, patient care, and education, it may be important in the future to have departments with individual faculty members more focused on selected components of the academic mission.

With respect to physician scientists, if we want to train young physicians for a career as basic investigators, we must make them competitive for peer review funding. For a physician to compete with Ph.D. investigators in similar areas, they must spend the majority of their time engaged in scientific re- 
search. Physician scientists in clinical departments will have some, albeit limited, involvement in clinical activities, while their Ph.D. colleagues will not. The advantage of having a clinical perspective applied to research activities often outweighs the disadvantage of having less than $100 \%$ effort devoted to research. If the amount of time devoted to research becomes substantially less than their Ph.D. colleagues, then the physician scientist will have difficulty competing for research dollars.

Then how should we train these future physician scientists? Since they will embark on a career in fundamental research, they must have rigorous training in the methodology to carry out hypothesis driven research. Our Ph.D. colleagues in basic science departments typically spend five to seven years in graduate school and oftentimes complete more than one postdoctoral fellowship before assuming a faculty position. An extended period of time is needed to allow a trainee to mature into an independent investigator, and it is naive to expect that we can train competitive physician scientists in significantly less time. Many training programs allow trainees to conduct basic research either prior to, or following, their core clinical training. If the trainee enters into basic research training prior to clinical training, then he or she must cease basic investigation while finishing clinical training. Very few research fields stay static while a trainee takes time out for clinical training. This is a good place to apply the litmus test that we discussed earlier. Would you add someone to your faculty and provide them with the space and support necessary to launch their career if they had not been in the research arena for one or more years? Will they be able to acquire independent peer-reviewed research support within a defined period of time? I don't find that I can answer these questions in the affirmative. Thus it may be desirable to have prospective physician scientists complete the clinical portion of their training first and then embark on basic research training, allowing for a smooth transition from mentored to independent research.

With respect to the content of research training, it is important that there is rigorous attention to the fundamentals of science. Most of our physician scientists receive their formal basic science instruction six or more years prior to the initiation of their research training. Thus re-training in these fundamentals is an imperative. An attractive approach is for the physician scientist to enroll in a formal Ph.D. training program after the completion of clinical training.

The second training pathway is that for developing clinician investigators. Similar to the established physician scientists of today, many of those currently engaged in clinical research have had no formal training in this discipline. Again, if we want to have the greatest chance of ensuring trainees' success in a clinical research career, we must provide them with the necessary formal training for this career path. Although the myth of "protected time" is still discussed by some, it is clear that in the future one must be able to fund the time that one spends in any type of research, including clinical research. Therefore young faculty members need to be positioned to be fundable with respect to their clinical research studies. How can we position our trainees for this expectation? Some training programs endeavor to train all of their fellows in a basic research laboratory, with the thought that formal training in the scientific method, whether it be in basic or clinical research, is adequate. I would argue that although better than no research training at all, basic research training does not adequately position one to embark on a career in clinical research. The clini- cal investigator trainee requires formal training in epidemiology, biostatistics, outcomes analysis, and/or health services research. It is likely that the most attractive trainees to pass the litmus test for hire as clinical investigators will be ones who have completed formal and rigorous training in clinical research methodology, quite possibly culminating in the MPH, Master's, or Ph.D. degree.

These formal training programs are becoming available in many institutions, and those who successfully complete these programs are finding it possible to become funded clinical investigators. A subset of those training to be clinical investigators may also go on to subspecialty clinical training. A trainee who has acquired both the tools to conduct clinical research and rigorous subspecialty clinical training will have the ability to formulate relevant questions and provide answers that will influence the practice of medicine.

The third broad type of training program to prepare one for a career in academic medicine is a training program for clinical educators. Similar to the aforementioned training programs, in the past it has been tacitly assumed that if one is trained to deliver high quality sophisticated clinical care, then that prepares one to teach others to do the same. It must be recognized that medical education is a sophisticated discipline in itself, and if a trainee chooses a career as a medical educator, and this is certainly a valid career path, then one should be formally trained to take this academic track. Part of the advanced residency or fellowship program for these trainees should be devoted to formal education in the methodology of teaching and the assessment of learning. At present, very few academic medical centers offer such a formal training program as a clinical educator.

Once we have helped a trainee successfully navigate through one of the three training tracks that I have described, and a young person joins the faculty at our academic institution, how do we ensure that they will be successful faculty members who both meet our expectations, and have their expectations met? Although each new faculty member will likely participate to some degree in all three components of the academic mission, that is research, education, and patient care, it may be even more important in the future for each faculty member to identify a specific academic focus. Protected time, like the triple threat, may be a relic of the past. If so, then it will be essential that we articulate clearly the expectations that we have for our academic faculty members. I believe it would be a mistake to lead new faculty members to believe that the goal of national prominence in research, education and patient care, is an attainable goal. As previously indicated, success as either a fundamental or clinical researcher will likely demand a singular effort focused on attaining this goal. Likewise, the master educator may also have to devote him or herself to continuing growth in this arena and regular and in-depth involvement in the educational process. Finally, the sophisticated clinical medicine of the type practiced in the academic medical center of today is not an activity that lends itself any longer to "part time doctors." In a referral-based academic medical center, one must demonstrate that the physicians caring for patients have something to offer above and beyond that of the superbly trained community practitioner. Those academic physicians who must limit their clinical involvement because of their focus on basic and clinical research, may not be viewed either by their colleagues or referring physicians as having a primary interest in providing state of the art clinical care. 
Thus four discrete faculty tracts may need to emerge to allow academic medical centers to successfully compete for research funding, to train academic physicians, and to deliver high quality and innovative clinical care. Three of the clinical tracks follow the previously described training programs. The physician scientist must expect to fund their salary for their research time and their research operations. Since these physician scientists perform activities almost identical to faculty members in basic science departments, they should also expect that their personal compensation be similar to faculty members in basic science departments. The physician scientists should expect in return, adequate research space and core support to foster their research programs. They should also expect a portion of their salary to be funded similar to the guarantees made to faculty members in basic science departments in some institutions. This would be a departure from many academic medical centers, where physician scientists in clinical departments are expected to be supplemented exclusively from clinical revenues. The faculty members should not be expected to participate in longitudinal direct patient care, nor be available for receiving referral patients at times other than certain assignments to the teaching service. This will allow these physician scientists to devote the majority of their time to developing their fundamental research programs, and in training the next generation of physician scientists.

The second faculty track is that of the clinician investigator. Again, these physicians should expect to derive support for the time that they devote to research, and should not expect to derive support for their research time from the clinical activities of other faculty members. If the institution provides support for a portion of the basic investigators time, then there should be similar expectations on the part of the clinician investigator. This may necessitate the redistribution of funds from those allocated to faculty members in basic science departments to investigators in clinical departments. Finally an institutional infrastructure must be in place to support the needs of patientoriented research.

A third faculty track is that of the clinical educator. These faculty members would be anticipated to provide for the majority of the clinical training of medical students, residents, and fellows. These physicians would be expected to generate a portion of their salary from clinical revenues, but would also expect to receive compensation for the clinical teaching that they perform. The faculty members in this track would also be expected to have received rigorous training in educational methodologies, and to be innovators with respect to clinical teaching. We must have clinical medicine taught by those trained to do so.

There is an additional faculty track that I have not described a training program for. This track would be the master clinician. By virtue of the core clinical training programs that all trainees in an academic medical center would complete, new faculty members in this area would have the training necessary to undertake this faculty track, and over time would acquire the clinical experience and sophistication to make them valuable members of the academic medical center. The physicians in this track might well devote the majority of their time to the practice of clinical medicine, with only a small portion of their time involved in clinical teaching or in clinical research. These physicians might expect to receive financial compensation similar to community physicians, and may not necessarily be members of the standing faculty. These physicians could provide for the patient care activities which are essential for maintaining a dynamic clinical enterprise, with their patients utilized to provide for the educational and research missions of the medical center. This activity is provided in some academic medical centers by "volunteer" physicians who are community practitioners. The challenge of the future is to integrate this type of physician into the full time activities of the academic medical center. It is important that the contribution of these physicians to the overall academic mission be viewed as valid and important, and that these physicians derive satisfaction in ways other than standard academic advancement.

The final challenge will be to integrate the different types of physicians in the academic medical center into multi-functional teams, perhaps focused in particular clinical areas. One might envision teams made up of a varying proportion of clinicians, clinical educators, clinician investigators, and physician scientists. The leader of the team could be a senior physician with demonstrated accomplishments in any one of the areas, but likely would be led by a clinician investigator or physician scientist. If constructed appropriately, the group would crossfertilize one another and supply each of the components of the academic mission. Patient care would be provided primarily by clinicians and clinical educators, to a varying extent by clinician investigators, and only to a minimum extent by the physician scientists. Clinical research would conducted primarily by the clinician investigator with participation of clinical educators, physician scientists, and clinicians. The basic research effort of the group would be primarily provided by the physician scientist members with some contribution from the other members of the team. The clinical teaching would be provided in large part by the clinical educators, again with some participation of other members of the group.

The concept of this team approach is that rather than having faculty members participate broadly in all components of the academic mission, each faculty member would have a primary responsibility for one aspect of the academic mission. It would be envisioned that these groups would be fluid, and that individual faculty members might participate in more than one group. This would be especially true for the physician scientists whose research interests might be relevant to a number of clinical areas. In the future it might be possible to recognize academic accomplishments through the success of the team as a whole. If the working group performed well, then individual team members would also receive recognition. In the end, the fundamental changes that are occurring in academic medicine may dictate that those in academic leadership positions recognize and encourage the building of multi-functional academic physician teams in addition to recognizing individual accomplishments.

In summary, the success of the academic enterprise is entering one of the most challenging episode in the history of academic medicine. If we are to preserve what we value in academic medicine, we are going to have to train the next generation to be competitive as academic physicians. We are also going to have to provide the necessary infrastructure for our basic and clinical research programs, and for our educational programs. These changes are essential to allow future faculty members a chance to experience the satisfaction that we have all derived from our choice of a career in academic medicine. 